\title{
Turbulent flow computation in a circular U-Bend
}

\author{
Abdelkrim Miloud $^{1}$, Mohammed Aounallah ${ }^{1, a}$, Mustapha Belkadi ${ }^{1}$, Lahouari Adjlout ${ }^{1}$, Omar Imine ${ }^{2}$ and Bachir Imine ${ }^{2}$ \\ ${ }^{1}$ Laboratory of Naval Aero-Hydrodynamics, Marine Engineering Department, Faculty of Mechanics, USTO \\ MB, P.O. 1505 EL-Mnaouar, Algeria. \\ ${ }^{2}$ Laboratory of Aeronautics and Propulsive Systems, Mechanical Engineering Department, Faculty of \\ Mechanics, USTO MB, P.O. 1505 EL-Mnaouar, Algeria.
}

\begin{abstract}
Turbulent flows through a circular $180^{\circ}$ curved bend with a curvature ratio of 3.375 , defined as the the bend mean radius to pipe diameter is investigated numerically for a Reynolds number of $4.45 \times 10^{4}$. The computation is performed for a U-Bend with full long pipes at the entrance and at the exit. The commercial ANSYS FLUENT is used to solve the steady Reynolds-Averaged Navier-Stokes (RANS) equations. The performances of standard $k-\varepsilon$ and the second moment closure RSM models are evaluated by comparing their numerical results against experimental data and testing their capabilities to capture the formation and extend this turbulence driven vortex. It is found that the secondary flows occur in the cross-stream half-plane of such configurations and primarily induced by high anisotropy of the cross-stream turbulent normal stresses near the outer bend.
\end{abstract}

\section{Introduction}

Over the last ten years, several numerical studies of fully developed curved-pipe flows are carried out to elucidate the problem of secondary flow patterns in such flows. On the other hand, a substantial amount of experimental information has been obtained for developing turbulent flows in curved ducts [1]. As is known in the literature, the secondary motions in the cross-stream half-plane of developing curved-pipe flows are influenced by different parameters. Among the more important are the curvature ratio, defined as the pipe radius over bend radius of curvature, Reynolds number, defined with respect to pipe diameter and centerline velocity, inlet flow distributions and above all by the condition of the entrance flow, ie; laminar or turbulent [2]. Azzola et al. [3] investigated turbulent curved-pipe flows with different curvature ratio and $\mathrm{Re}$ and fully developed turbulent flow at the inlet. The total and circumferential velocity measurements indicated the presence of a center cell near the pipe center witch is confirmed further by the $\mathrm{k}-\varepsilon$ model calculations of Azzola et al. [4] and Anwer et al [5]. In both these calculations, radial velocity component reversal along the horizontal diameter near the pipe center is found. However, secondary flow separation near the inner bend is not observed. This is a consequence of the secondary boundary layer being turbulent and, therefore, can overcome a larger pressure gradient before separation. As a result, only two secondary cells are found in the cross- stream half-plane of turbulent curved-pie flows. It is known that Reynolds-stress can generate vorticity in a turbulent flow. The vertical motion thus generated is classified as Prandtl's secondary flow of the second kind and is referred to as turbulence-driven or stress-induced secondary flow by Bradshaw [6]. This interesting phenomenon is a unique feature of turbulent flow.

From the literature survey, it is found experimentally difficult and expensive to investigate secondary motions in curved-pipe flows, and alternative is to study them numerically. It should be borne in mind that, even in numerical computation, resolution of secondary cells is affected by many factors. Among the more important ones are numerical technique, grid size, and turbulence models. In order to gain an impression of how successful or otherwise the widely used $k-\varepsilon$ eddy viscosity model and the RSM are in predicting this flow behavior, the solving scheme developed has provided the basis for a numerical simulation. The objectives of this study are to investigate the effect of the turbulence models: the k- $\varepsilon$ and the RSM models. Their performances are evaluated by comparing their numerical results against experimental data and testing their capabilities to capture the formation and extend this turbulence driven vortex.

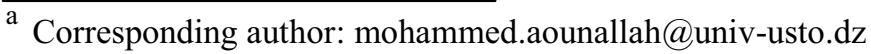




\section{Problem position}

The basic components of the flow test section are shown schematically in Fig. 1. The configuration tested is composed with two straight pipes and a $180 \mathrm{deg}$. curved pipe. The pipe cross-section was circular throughout with a $4.45 \mathrm{~cm}$ inner diameter $(D)$. The ratio of bend mean radius of curvature to pipe diameter was $R c / D=3.375$. Both tangents were of length $X=54.7 D$, being, respectively, attached to the $0 \mathrm{deg}$. (inlet) and $180 \mathrm{deg}$. (outlet) planes of the bend by means of flanges.

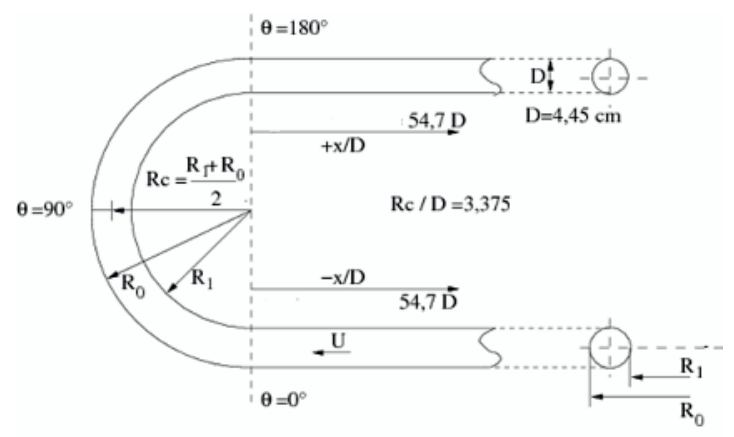

Figure 1. U-Bend geometry features

\section{Governing equations}

The viscous incompressible turbulent flow is described by Navier-Stokes equations completed by one of the two turbulence models: the standard $\mathrm{k}-\varepsilon$ and the Reynolds stress model RSM. The mean flow pressure is $P$ and the mean velocity $u_{i}$.

$$
\begin{aligned}
& \frac{\partial U_{j}}{\partial x_{j}}=0 \\
& \frac{\partial}{\partial x_{j}}\left(\rho u_{j} u_{i}\right)=-\frac{\partial P}{\partial x_{i}}+ \\
& \frac{\partial}{\partial x_{j}}\left[\left(\mu+\mu_{t}\right)\left(\frac{\partial u_{i}}{\partial x_{j}}+\frac{\partial u_{j}}{\partial x_{i}}\right)\right]-\frac{2}{3} \frac{\partial}{\partial x_{j}}\left(\mu_{t} \frac{\partial u_{j}}{\partial x_{j}}\right)
\end{aligned}
$$

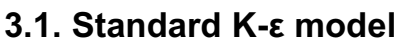

$$
\begin{aligned}
\frac{\partial}{\partial x_{j}}\left(\rho k u_{j}\right) & =\frac{\partial}{\partial x_{j}}\left[\left(\mu+\frac{\mu_{t}}{\sigma_{k}}\right) \frac{\partial k}{\partial x_{j}}\right]+G_{k}-\rho \varepsilon \\
\frac{\partial}{\partial x_{j}}\left(\rho \varepsilon u_{j}\right)= & \frac{\partial}{\partial x_{j}}\left[\left(\mu+\frac{\mu_{t}}{\sigma_{\varepsilon}}\right) \frac{\partial \varepsilon}{\partial x_{j}}\right] \\
& +C_{1 \varepsilon} \frac{\varepsilon}{k} G_{k}-C_{2 \varepsilon} \rho \frac{\varepsilon^{2}}{k}
\end{aligned}
$$

$$
\mu_{t}=\rho C_{\mu} \frac{k^{2}}{\varepsilon}
$$

$G_{k}$ represents the production of turbulence kinetic energy due to the mean velocity gradients and the constants are: $\mathrm{C}_{1 \varepsilon}=1.44, \mathrm{C}_{2 \varepsilon}=1.92, \mathrm{C}_{\mu}=0.09, \sigma_{\mathrm{k}}=1.0, \sigma_{\varepsilon}=1.3$

\subsection{Reynolds Stress Model RSM}

$$
\begin{aligned}
& \frac{\partial}{\partial t}\left(\rho \overline{u_{i} u_{j}}\right)+\frac{\partial}{\partial x_{k}}\left(\rho U_{k} \overline{u_{i} u_{j}}\right)= \\
&\left.-\frac{\partial}{\partial x_{k}}\left[\rho \overline{u_{i} u_{j} u_{k}}+\overline{p\left(\delta_{k j} u_{i}+\delta_{i k} u_{k}\right)}\right)\right] \\
&+D_{L, i i}+P_{i j}+\phi_{i j}-\varepsilon_{i j} \\
& \frac{\partial}{\partial t}(\rho k)+ \frac{\partial}{\partial x_{j}}\left(\rho k U_{j}\right)= \\
& \frac{\partial}{\partial x_{j}}\left[\left(\mu+\frac{\mu_{t}}{\sigma_{k}}\right) \frac{\partial k}{\partial x_{j}}\right]+\frac{1}{2} P_{i i}-\rho \varepsilon \\
& \frac{\partial}{\partial t}(\rho \varepsilon)+\frac{\partial}{\partial x_{j}}\left(\rho \varepsilon U_{j}\right)= \\
& \frac{\partial}{\partial x_{j}}\left[\left(\mu+\frac{\mu_{t}}{\sigma_{\varepsilon}}\right) \frac{\partial \varepsilon}{\partial x_{j}}\right] \\
&+C_{1 \varepsilon} \frac{1}{2} P_{i i} \frac{\varepsilon}{k}-C_{2 \varepsilon} \rho \frac{\varepsilon^{2}}{k} \\
& \mu_{t}=\rho C_{\mu} \frac{k^{2}}{\varepsilon}
\end{aligned}
$$

$D_{L, i j}, P_{i j}$ do not require any modeling, its represent the molecular diffusion and the stress production respectively. However, $\phi_{i j}$ and $\varepsilon_{i j}$ represent the pressure strain and the dissipation and need to be modeled to close the equations. The constants are: $\mathrm{C}_{1 \varepsilon}=1.0, \mathrm{C}_{2 \varepsilon}=1.92, \mathrm{C}_{\mu}$ $=0.09, \sigma_{\mathrm{k}}=0.82, \sigma_{\varepsilon}=1.0$

\section{Grid generation}

Figure 2 shows the curvature of the U-bend of the physical domain meshed into 391950 hexahedral control volumes. On the other hand, the region close to the wall is divided into smaller hexahedral cells to avoid the numerical error in this region. The geometry and the grid are generated using Gambit preprocessor taking into account the boundary layer refinement with 6 layers near the walls of the two pipes. The height of the first cell is calculated through the estimating value of $y^{+}$which guaranteed the using of a high Reynolds number model of turbulence. The mesh quality is excellent: $41 \%$ of total cells have an equisize skew coefficient bellow than 0.1 and $26 \%$ between 0.1 and 0.2 . The skew of the remaining cells do not exceed 0.5 . 


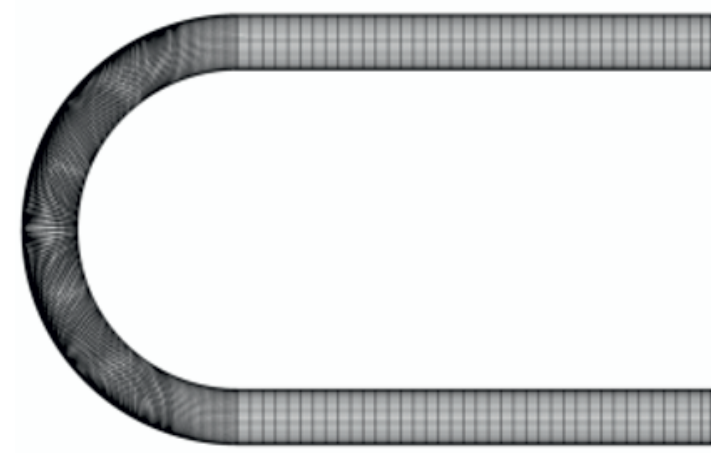

Figure 2. The hexahedral grid of the U-Bend

\section{Numerical approach}

The problem treated is a steady three-dimensional turbulent flow through a circular $180^{\circ}$ curved bend with a curvature ratio of 3.375. The Reynolds number based on the velocity at the centreline and the diameter of the pipe is fixed to $4.45 \times 10^{4}$. All the simulations are done with the commercial code: ANSYS Fluent 6.3.26. The segregated implicit solver based on the pressure is used to solve the governing equations of motion. The algorithm Simple is used for coupling pressure-velocity. The full simulations were conducted the $2^{\text {nd }}$ order upwind. The solution converges when the residuals fall down $10^{-10}$ for continuity, momentum and turbulence quantities.

\section{Results and discussion}

Figure 3 shows the position of the section where the contours and the profiles of velocity are plotted. The section 1,2 and 8 are set in the straight pipes following the $\mathrm{z}$ axis while sections $3,4,5,6$ and 7 are located in the curved part with the appropriate angles showed in the figure.

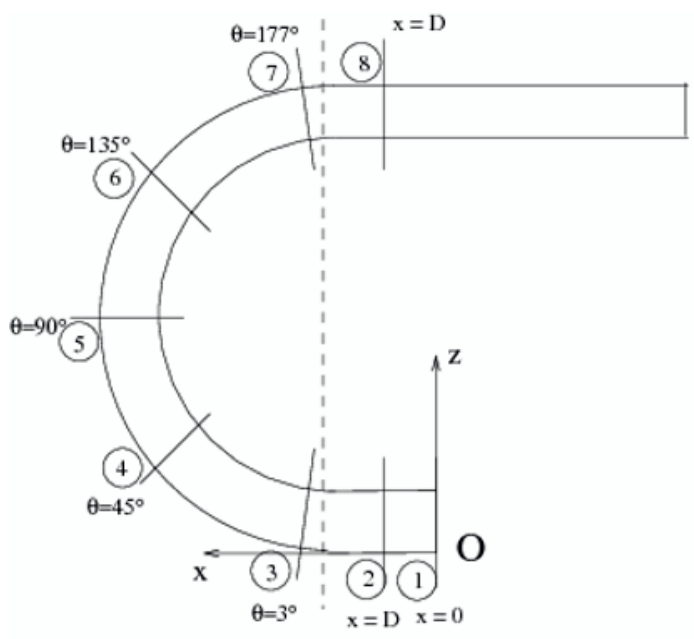

Figure 3. Location of the reference section
Figure 4 shows the wall $\mathrm{y}+$ distribution on the curved part of the U-Bend obtained by both turbulence models. The range of the $y+$ is obviously adequate with using a high Reynolds number turbulence model. This figure illustrates that the resolution of the grid used is satisfactory with the turbulence model type. Commonly, the two turbulence models predict a largest $y+$ in the inside region and smallest $\mathrm{y}^{+}$in the outside of the $\mathrm{U}$ Bend. A clear quantitative difference exists between the predictions of the two models tested mainly in the curved part. This difference is certainly due to difference in the velocity field predicted.

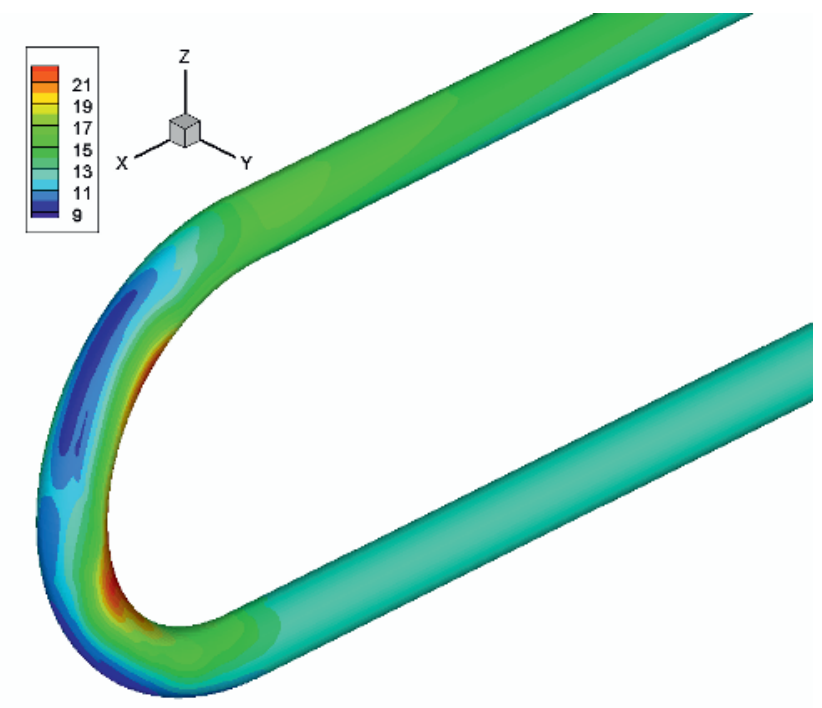

a) $\mathrm{k}-\boldsymbol{\varepsilon}$ model

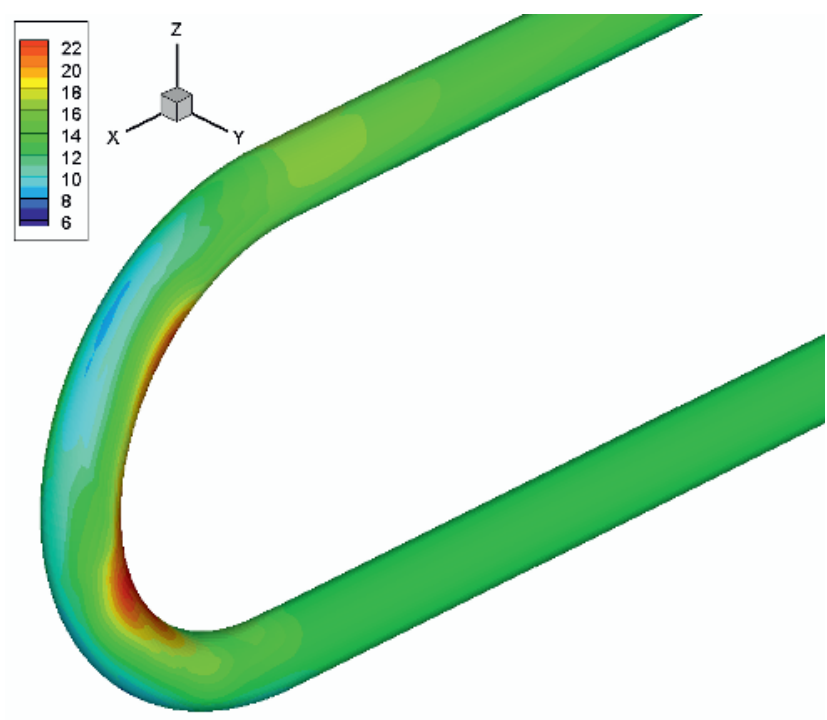

b) RSM model

Figure 4. Wall $\mathrm{y}+$ distribution on the U-Bend 
Figure 5 shows the normalized flow velocity (stream wise direction) at different sections obtained with both turbulence models, the velocity is normalized by the reference velocity taken in definition of the Reynolds number, $4.45 \times 10^{4}$. Scans are made at $X / D=0$ and 1 in the straight pipes and $\theta=3,45,90,135$, and $177 \mathrm{deg}$ in the bend. The contours are shown as the left part indicates the inside of the tube and the right part designates the outside one. The computed longitudinal velocity component at the section $2, X / D=1$ is identical between both simulations. The forms of the contours are accurately circular and show good agreement with the developed in a straight pipe. At this position, the flow is fully developed in the central region. Close to the wall, the longitudinal velocity tends to zero in respect with the adherence condition.

In the first half of the bend ( $\theta=3$ to $90 \mathrm{deg})$ as is shown in the section 4 , the streamwise velocity contour show a strong deformation of circular forms obtained previously but the features is still symmetric following the horizontal radial axis. The bulk velocity contours tend to be longer in the vertical direction and less large in the horizontal direction. Close to the inside region of the curved pipe, the flow tend to accelerate and contrary to the previous section, notable qualitative discrepancies exist between both predictions obtained by the turbulence models.

The occurrence of a second cross-stream flow reversal past $\theta=90 \mathrm{deg}$ in the bend shows a large part dominated by an accelerated flow except the region close to the inside of the curved pipe. From the whole of the contours presented, it is clear seen that location characterizes the maximum reached, that why the surface of the bulk velocity decreases in the coming positions. At $\theta=135$ $\mathrm{deg}$, the figure shows the core of the streamwise flow neighboring the inside part losing speed while the flow near the inside wall accelerates. According the last section $X / D=1$ in the outlet branch of the U-Bend, the flow seem to be accelerated in the half vertical section close to the outside part of the curved pipe. On the other half, the streamwise velocity flow decreases especially near the inside region.

To get more quantitative agreement of the computation, the numerical profiles of the streamwise obtained by both models are compared with the experimental data of Azzola et al. [3] for the different locations cited. A good quantitative agreement is obtained by both turbulence model simulations for the first sections, $\mathrm{X} / \mathrm{D}=1$ and until $\theta=3$, no great curvature of the U-bend. The numerical results shows a slight over estimation for $\theta=45 \mathrm{deg}$ where the increase of the circumferential component of mean velocity at corresponding station reveal the development of a strong secondary flow. At the middle section of the U-Bend, $\theta=$ 90 , both turbulence models underestimates the stream wise velocity in the first half and over predicts on the second half. At $\theta=135$, the numerical results seem to be more close to the experimental data, a slight difference
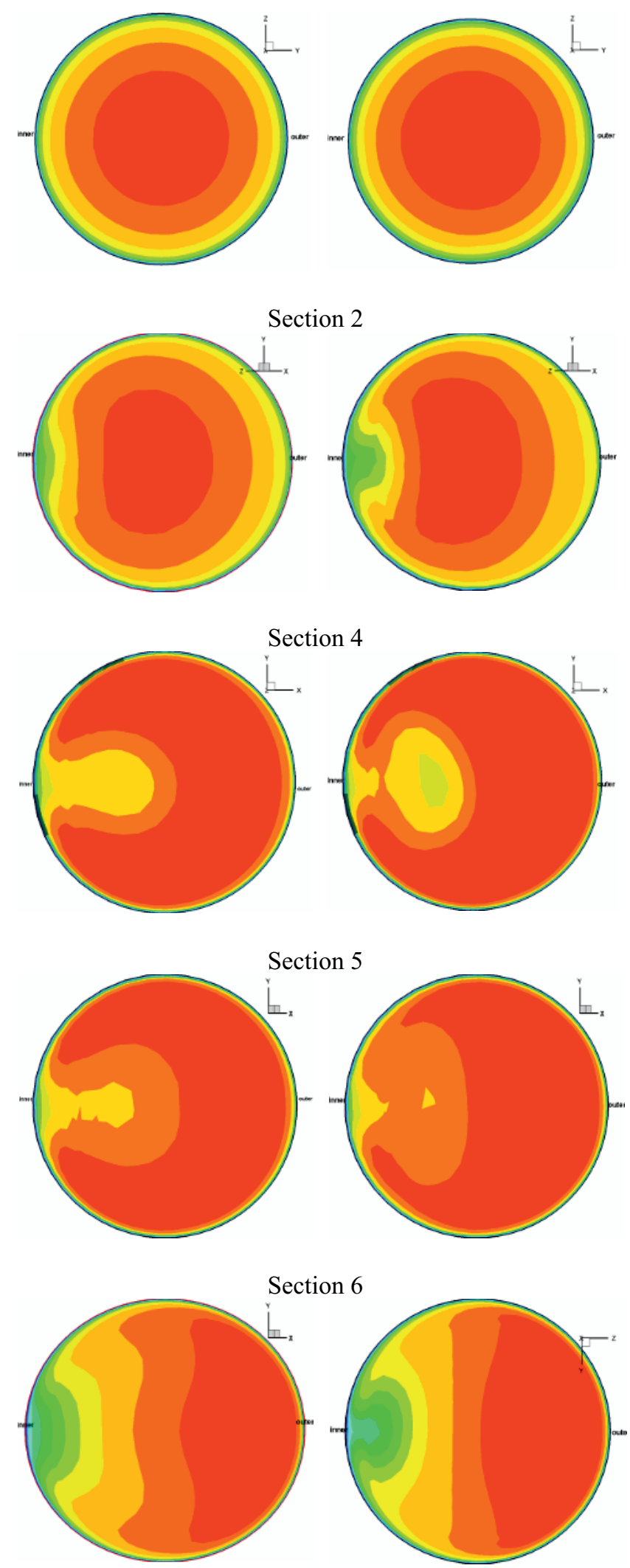

Section 8

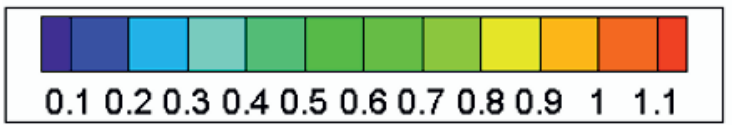

$k \sim e$ model

RSM model

Figure 5. Normalized flow velocity at different sections 

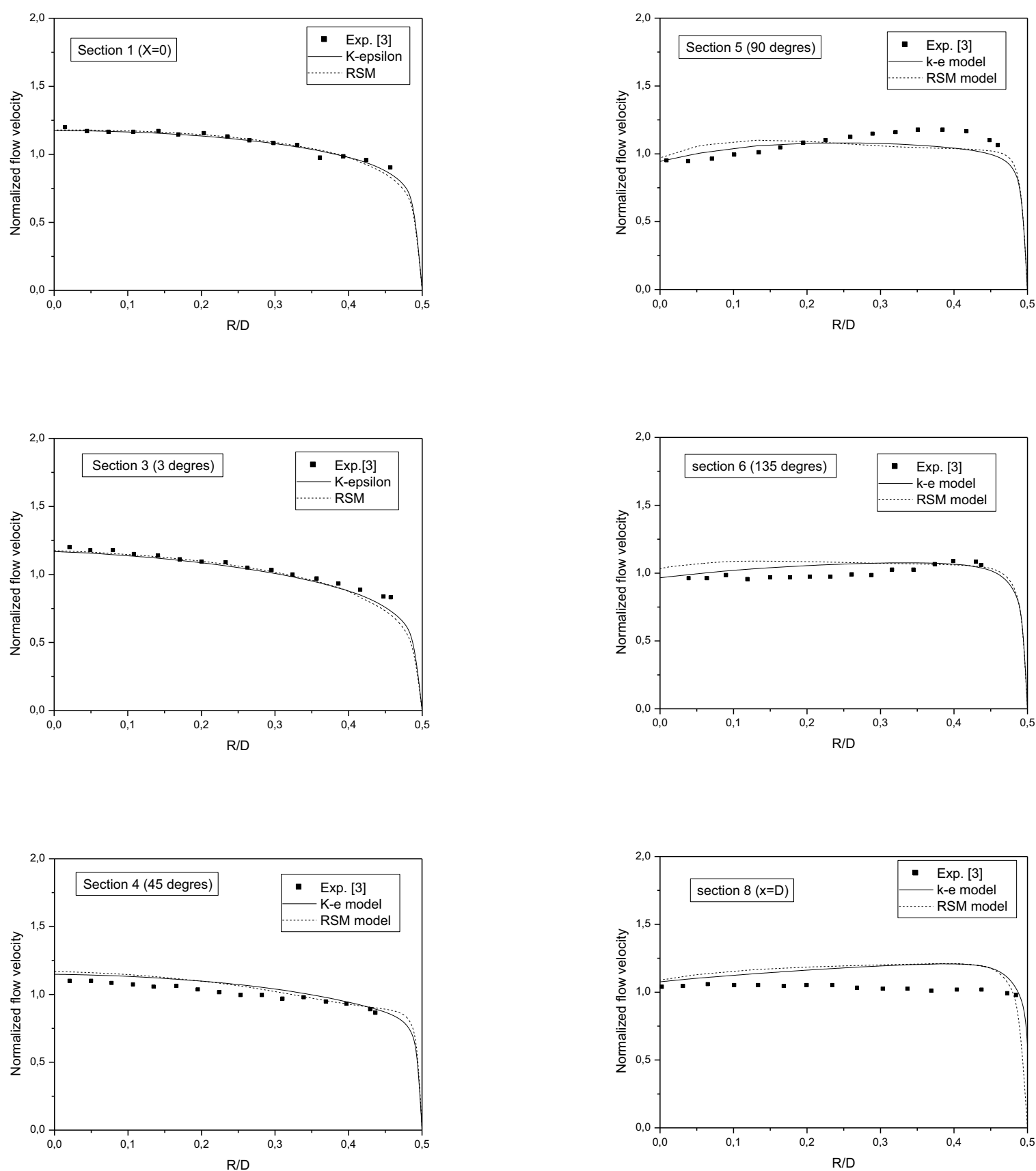

Figure 6. Normalized flow velocity for different sections

appear at the central part, while a major discrepancy is shown at the last section $X / D=1$ in the out coming branch.

Figure 7 shows the secondary flow vectors in the cross-sectional plane. The increase of the circumferential component of mean velocity at corresponding station reveals the development of a strong secondary flow. The secondary flow is induced by the transverse pressure gradient set up between the outer and inner wall regions of the bend. In the pipe center, it works to overcome and reverse the sense of the cross-stream motion in the inlet flow. Each symmetrical half section of the bend develops two counter-rotating vortical structures. The more intense of the two, located between the pipe wall and the core of the flow, preserves the sense of cross-stream motion induced by the transverse pressure gradient at the start of the bend. The smaller, weaker, structure is mainly confined to the core and is attributed to the formation of a transverse pressure gradient opposite in sign to that at the start of the bend. 


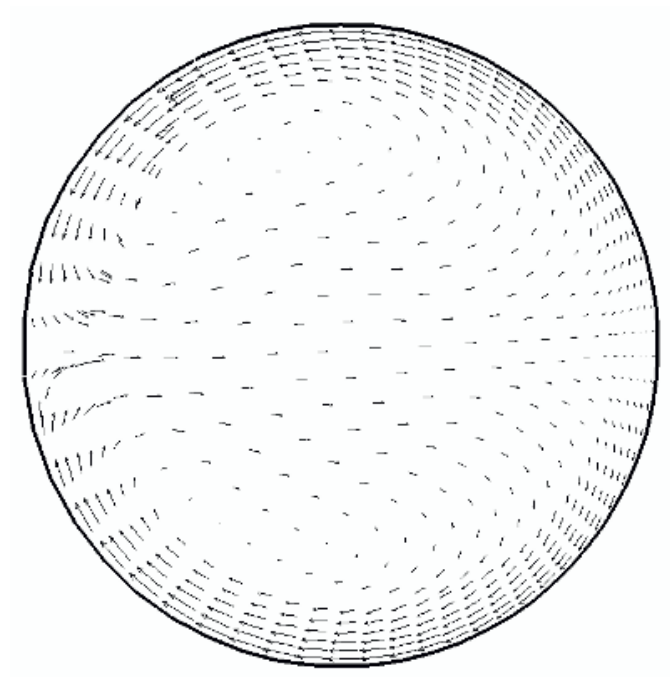

Figure 7. Cross-stream vector at the section $\theta=90 \mathrm{deg}$.

\section{Conclusion}

In order to investigate the performance of the turbulence models: the widely used $\mathrm{k}-\varepsilon$ eddy viscosity and the second order closure RSM to predict reasonably the flow behavior in a $180 \mathrm{deg}$ U-Bend curved pipe at a pipe Reynolds number of $4.45 \times 10^{4}$ and a radius ratio of and a radius ratio of 3.375 has been carried. The numerical study reveals that the secondary flow pattern in a curved pipe is very complicated. The results support the notion of an additional (symmetrical) pair of counter-rotating vortical structures embedded in the core of the flow within the curved pipe.
The numerical simulations have reproduced with a gratifying degree of fidelity the measured evolution of the flow with some slight quantitative discrepancies in the curved part of the U-Bend. No significant differences are detected between the two turbulence models tested.

\section{References}

1. J. A. C Humphrey, J. H Whitelaw and G. Yee, J. Fluid Mech., Vol. 103, 443-463 (1981).

2. Y.G. Lai, R.M.C. So and H.S. Zhang, Theoret. Comput. Fluid Dynamics, Vol. 3, 163-180 (1981)

3. J. Azzola and J. A. C Humphrey, Developing Turbulent Flow in a $180^{\circ}$ Curved Pipe and its Downstream Tangent, Report LBL-17681, Materials and Molecular Research Division, Lawrence Berkeley Laboratory, University of California (1984).

4. J. Azzola, J. A. C Humphrey, H. Iacovidas and B.E. Launder, J. Fluids Engrg. 108, 214-221 (1986).

5. M. Anwer, R.M.C. So and Y.G. Lai, Phys. Fluids A 1, 1387-1397 (1989).

6. P. Bradshaw, Ann. Rev. Fluid Mech. 19, 53-74 (1987). 